\title{
Effects of Hypoxia on Respiratory Neural Output and Lower Esophageal Sphincter Pressure in Piglets
}

\author{
PAKAPHAN KIATCHOOSAKUN, ISMAIL A. DRESHAJ, JALAL M. ABU-SHAWEESH, \\ MUSA A. HAXHIU, AND RICHARD J. MARTIN \\ Department of Pediatrics, Case Western Reserve University, Rainbow Babies and Children's Hospital, \\ Cleveland, Ohio 44106-6010, U.S.A. [I.A.D., J.M.A.-S., M.A.H., R.J.M.]; Department of Physiology and \\ Biophysics, Howard University, Washington, D.C. 20059, U.S.A. [M.A.H.]; Department of Pediatrics, \\ Faculty of Medicine, Khon Kaen University, Khon Kaen 40002, Thailand [P.K.]
}

\begin{abstract}
ABSTR
We have previously documented anatomic and functional
relationships between ventilatory and autonomic neural output.
Therefore, we hypothesized in this study that hypoxia-induced
changes in respiratory neural output are associated with changes
in autonomic regulation of lower esophageal sphincter (LES)
pressure. Respiratory neural output, heart rate, and LES pressure
were measured before and during a 3-min exposure to $8 \%$
oxygen (balance nitrogen) in 123 - to 7-d-old piglets. Respiratory
neural output was determined from diaphragmatic electromyo-
gram and LES pressure from an esophageal catheter. Studies
were repeated after atropine administration in eight animals.
Hypoxic exposure resulted in significant increases in diaphrag-
matic amplitude, respiratory rate, and minute diaphragmatic
activity as well as heart rate. The biphasic response of diaphragm
amplitude peaked at 1 min, whereas the responses of respiratory
frequency and heart rate were sustained. Hypoxia caused a 50\%
\end{abstract}
Neuroanatomic studies have identified that neuronal circuits involved in coordination of respiratory control are linked to parasympathetic motor output from vagal preganglionic neurons in the brainstem $(1,2)$. A functional role for such interconnections has been documented in several species, including puppies and piglets $(3,4)$. For example, hypoxic stimulation in piglets is associated with an increase in both phrenic neural output and contractile responses in peripheral airways and/or lung parenchyma, which are cholinergically mediated (5). This is consistent with the clinical observation that an acute reduction in inspired oxygen may lead to airway constriction in infants with chronic lung disease (6).

Received September 7, 2001; accepted February 8, 2002.

Correspondence and reprint requests: Richard J. Martin, M.D., Rainbow Babies and Children's Hospital, Division of Neonatology, 11100 Euclid Avenue, Cleveland, OH 44106-6010, U.S.A.; e-mail: rxm6@po.cwru.edu

Supported by National Institutes of Health Grant HL62527, and the Ross Products Division of Abbott Laboratories. increase in LES pressure $(p<0.05)$, which was eliminated by i.v. atropine administration. Development of apnea during subsequent hyperoxic exposure was always followed by a decline in LES pressure. Hypoxia-induced increase in respiratory neural output and accompanying increase in heart rate are associated with enhanced constrictive output to the LES. Blockade by atropine implicates a peripheral cholinergic mechanism for this LES response. We speculate that whereas hypoxia in the presence of enhanced respiratory neural output seems to be protective against reflux, decreased respiratory drive and accompanying apnea may be associated with a decline in LES tone and predispose to gastroesophageal reflux. (Pediatr Res 52: 50-55, 2002)

Abbreviations
LES, lower esophageal sphincter
DMV, dorsal motor nucleus of the vagus

Vagal preganglionic neurons, including neurons of the DMV, also contribute preganglionic parasympathetic motor innervation to the LES (7). Therefore, alterations in respiratory neural output elicited by chemoreceptor stimulation might be expected to modulate LES pressure. This would be of particular interest during early maturation, as recurrent apnea with resultant desaturation and gastroesophageal reflux are both common in premature infants, although their relationship is controversial $(8-11)$ and difficult to simulate in an animal model. In the current study, we therefore used a piglet model to test the hypothesis that hypoxia-induced changes in respiratory neural output would be associated with changes in autonomic regulation of LES pressure.

\section{MATERIALS AND METHODS}

Experimental preparation. All experiments were conducted in accordance with institutional guidelines and were approved by the Institutional Animal Care and Use Committee at Case 
Western Reserve University. Experiments were performed in 12 newborn piglets of either sex, aged 3-7 d and weighing $1.6-3.2 \mathrm{~kg}$. The piglets were fasted for $12 \mathrm{~h}$ before each experiment.

Piglets were initially sedated using a mixture of intramuscular ketamine hydrochloride $(14 \mathrm{mg} / \mathrm{kg})$ and xylazine $(2.8$ $\mathrm{mg} / \mathrm{kg}$ ), then anesthetized with an i.v. mixture of $\alpha$-chloralose $(24 \mathrm{mg} / \mathrm{kg})$ and urethane $(120 \mathrm{mg} / \mathrm{kg})$, such that the animals were unresponsive to stimuli, yet breathing spontaneously. After a high cervical tracheostomy, the piglets inspired $100 \%$ oxygen through a T-piece. A femoral artery was catheterized for continuous monitoring of heart rate and blood pressure. A cephalic vein was cannulated to allow for continuous administration of fluid to maintain hydration as well as for administration of drugs. Studies were performed with the piglets placed on a heating pad to maintain body temperature between $37^{\circ}$ and $38^{\circ} \mathrm{C}$.

Diaphragmatic electromyographic (EMG) signals were measured by a bipolar electrode consisting of two stainless steel hook wires, spaced 2-3 $\mathrm{mm}$ apart, implanted under direct vision into the costal part of the diaphragm. The diaphragmatic electrical potentials were amplified using a Grass amplifier (model P511 K; Grass Instruments, Quincy, MA, U.S.A.) and then processed by a Paynter filter integrated with a time constant of $100 \mathrm{~ms}$ to obtain a moving average signal. The electrical wires were led through the abdominal incision and the animal's abdomen was sutured closed.

Manometric technique. A micromanometric assembly incorporating a sleeve sensor (Dentsleeve Pty. Ltd., Adelaide, Australia) was fed down the esophagus via the mouth. The manometric channels of the assembly were continuously perfused with degassed distilled water at $80 \mu \mathrm{L} / \mathrm{min} / \mathrm{channel}$. The assembly consisted of gastric and lower esophageal pressure ports. The sleeve sensor was placed at the LES by slowly withdrawing the catheter from within the stomach so that the high-pressure zone was monitored at the lower esophageal pressure port, and the adjacent side-hole recorded gastric pressure. Catheter location was confirmed postmortem at the conclusion of each study. The transducer calibration procedure comprised measurement of deflection of the pressure transducer signal generated by each $\mathrm{mm} \mathrm{Hg}$ pressure change, with a readily detectable change of $0.3 \mathrm{~mm} \mathrm{Hg}$. Stability of this system was always confirmed by application of a known resistance before and after each study. The experimental protocol was not started until at least $10 \mathrm{~min}$ had elapsed after placement of the catheter, to allow any reflexes triggered by its insertion to subside. An equilibration period of $15 \mathrm{~min}$ was allowed after each stimulus, or after administration of atropine in order that a steady basal level of sphincter pressure was reached.

Experimental protocol. Four channels were used to record simultaneous baseline values of diaphragmatic EMG, lower esophageal pressure, gastric pressure, and mean arterial pressure and heart rate while the animals were spontaneously breathing $100 \%$ oxygen. Hypoxic exposure with $8 \%$ inspired oxygen, balance nitrogen, then occurred for $3 \mathrm{~min}$. This resulted in a mean change in partial pressure of arterial oxygen $\left(\mathrm{PaO}_{2}\right)$ from $281 \pm 26$ to $24 \pm 2 \mathrm{~mm} \mathrm{Hg}$. After hypoxic exposure, the animals were returned to $100 \%$ inspired oxygen. Atropine methylnitrate, $1 \mathrm{mg} / \mathrm{kg}$, was then infused i.v. in eight of the 12 piglets and hypoxic exposure was repeated beginning 15 min after atropine administration. In five of these piglets, a second hypoxic exposure of 3-min duration was performed, however, these data were not included in the overall data analysis because of the small sample size.

Data analysis. Respiratory neural output was determined from amplitude of diaphragmatic EMG expressed in arbitrary units (AU), respiratory rate per minute, and minute diaphragmatic activity (amplitude $\times$ respiratory rate). LES pressure was expressed as the difference between recorded values of pressure at the lower esophageal high-pressure zone and gastric pressure at each time point. In assessment of the response to hypoxia, diaphragmatic amplitude, respiratory rate, minute diaphragmatic activity, heart rate, and LES pressure were analyzed over each 30-s interval.

Statistical analysis. All data are expressed as mean \pm SE. One-way ANOVA with repeated measures was used to test the effect of hypoxia on diaphragmatic amplitude, respiratory rate, minute diaphragmatic activity, heart rate, and LES pressure. A Newman-Keuls procedure was used for post hoc comparisons. Two-way ANOVA was used to test the effect of atropine administration on the LES pressure response to hypoxia. A $p$ value $<0.05$ was considered statistically significant.

\section{RESULTS}

Hypoxic exposure caused a significant increase in diaphragmatic amplitude, respiratory rate, and minute diaphragmatic activity as well as heart rate (all $p<0.0001$, one-way ANOVA). The increase in diaphragmatic amplitude peaked at 1 min after hypoxic exposure and then slightly decreased toward the latter part of the response. At peak response, amplitude increased by $61 \%$ from baseline $(p<0.01)$ (Fig. 1). The response of respiratory rate was significantly increased after $2 \mathrm{~min}$ and peaked at $3 \mathrm{~min}$ of hypoxic exposure. At peak response, respiratory rate increased by $36 \%$ from a baseline of $29.6 \pm 2.1$ breaths $/$ min to $40.2 \pm 2.7$ breaths $/ \min (p<0.01)$ (Fig. 1). The increase in minute diaphragmatic activity peaked at $1 \mathrm{~min}$ of hypoxic exposure and then plateaued toward the latter part of the response. At peak response, minute diaphragmatic activity increased by $79 \%$ from baseline $(p<0.01)$ (Fig. 1).

In response to hypoxic exposure, heart rate was significantly increased after $1 \mathrm{~min}$ and then increased continuously throughout the duration of hypoxia. At peak response, heart rate increased by $43 \%$ from a baseline of $136 \pm 9$ beats/min to 195 \pm 7 beats $/$ min $(p<0.01)$ (Fig. 1). Mean arterial pressure did not differ significantly at $3 \mathrm{~min}(83 \pm 3 \mathrm{~mm} \mathrm{Hg})$ when compared with control $(80 \pm 2 \mathrm{~mm} \mathrm{Hg})$.

Hypoxic exposure caused a significant increase in LES pressure $(p<0.05$, one-way ANOVA). LES pressure was significantly elevated at 2.5 and $3 \mathrm{~min}$ of hypoxic exposure compared with baseline. At peak response, LES pressure increased by $49 \%$ from a baseline of $4.9 \pm 0.7 \mathrm{~mm} \mathrm{Hg}$ to $7.3 \pm$ $0.9 \mathrm{~mm} \mathrm{Hg}(p<0.05)$ (Figs. 2 and 3). This was secondary to 

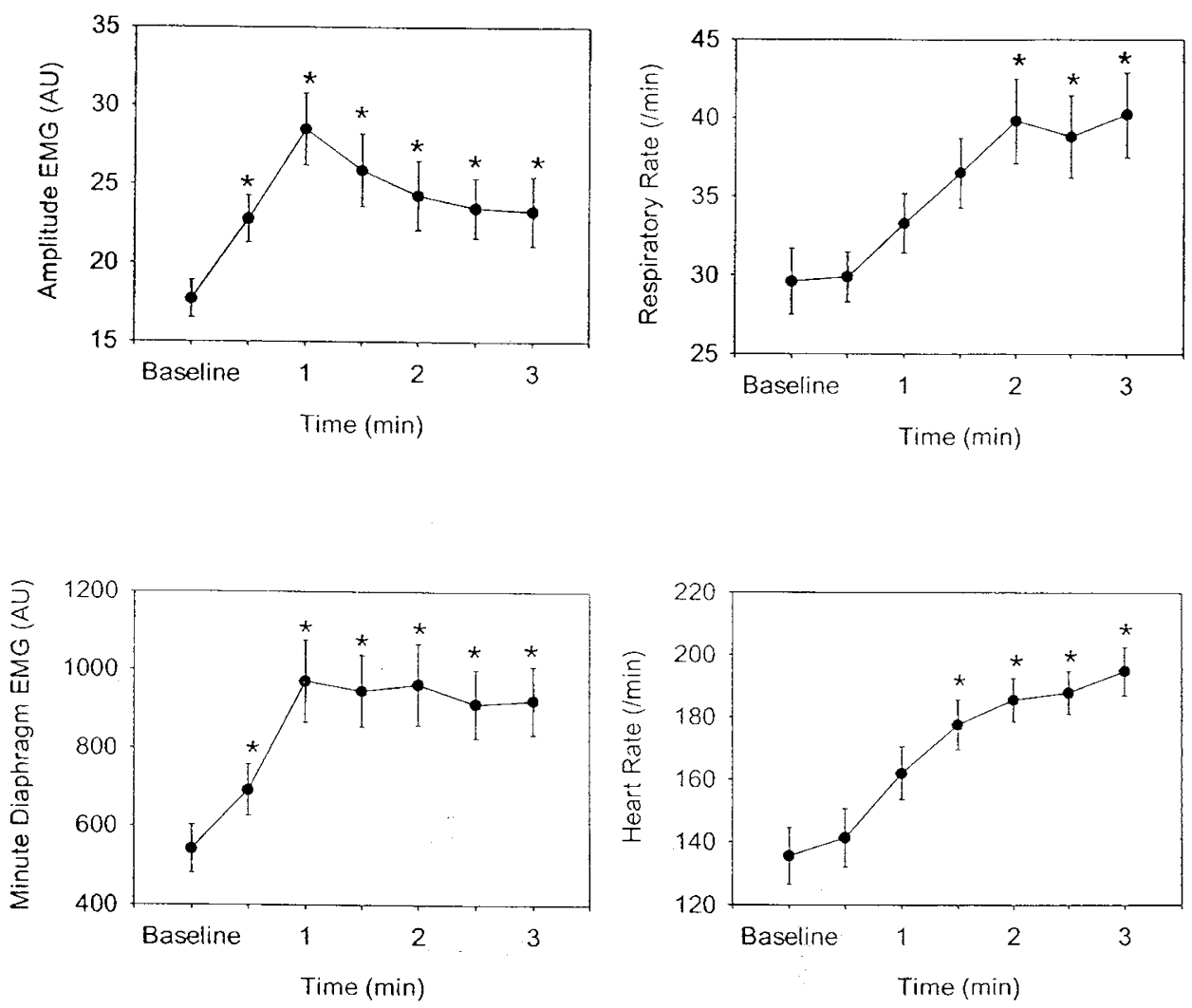

Figure 1. Hypoxic exposure caused an increase in diaphragmatic amplitude, respiratory rate, minute diaphragm activity, and heart rate in the 12 piglets. The amplitude response peaked at $1 \mathrm{~min}$ and then slightly declined toward the latter portion of the response. All responses were statistically significant via one-way ANOVA, and asterisks indicate a significant increase above baseline via Newman-Keuls procedure.

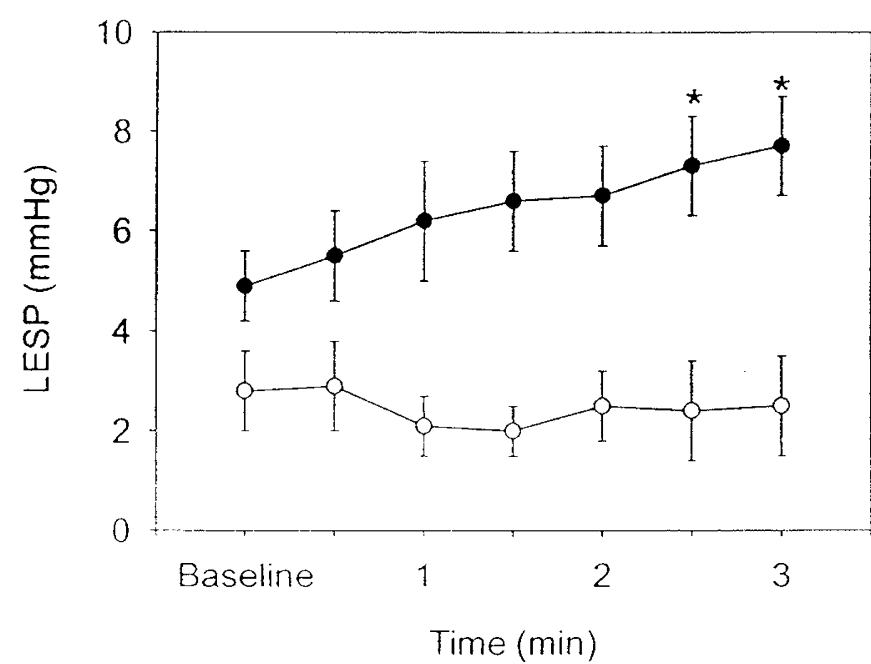

Figure 2. Hypoxic exposure caused a significant increase in lower esophageal sphincter pressure (LESP) (via one-way ANOVA and Newman-Keuls procedure, $n=12$, closed circles $)$. Atropine eliminated this increase in LESP $(n=$ 8, open circles).

an increase in pressure at the lower esophagus, as there was no measurable change in gastric pressure.

Atropine administration $(n=8)$ resulted in a decrease in baseline LES pressure from $4.9 \pm 0.7$ to $2.8 \pm 0.8 \mathrm{~mm} \mathrm{Hg}$. Furthermore, atropine eliminated the hypoxia-induced increase in LES pressure ( $p<0.02$, two-way ANOVA) (Fig. 2), without affecting the respiratory response.
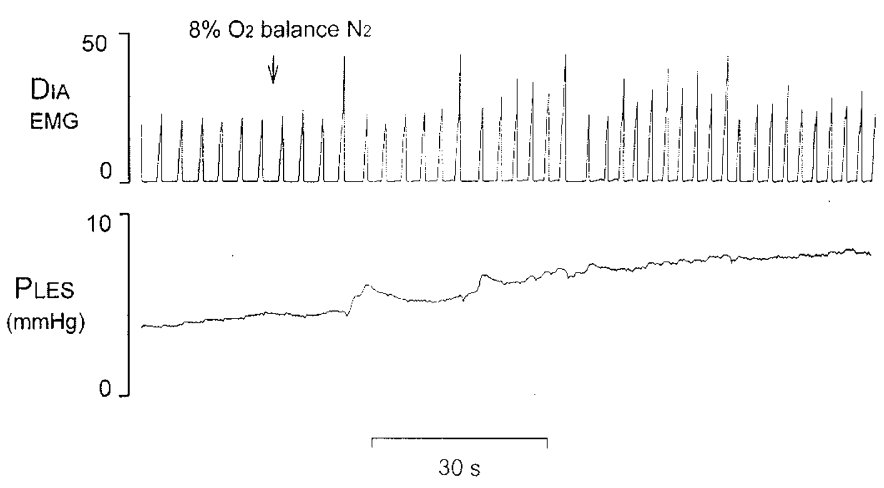

Figure 3. An example of the diaphragm (DIA) and LES pressure (Ples) responses to hypoxic exposure in a piglet. Peak integrated DIA EMG, expressed in arbitrary units; PLES, expressed in $\mathrm{mm} \mathrm{Hg}$; and respiratory rate all increased.

Re-exposure to $100 \%$ inspired oxygen after 3 min of a first hypoxic exposure was associated with apnea of $>10 \mathrm{~s}$ in 5 of 12 piglets. These apneic episodes began within $25 \mathrm{~s}$ of reintroducing $100 \%$ inspired oxygen. In five piglets who did not exhibit apnea after a first hypoxic exposure, a second hypoxic exposure was performed. Three piglets in this re-exposed group exhibited apnea, which commenced before reintroduction of $100 \%$ oxygen, as seen in Figure 4. Therefore, apnea was observed in a total of eight animals. Apnea was always followed by a decrease in LES pressure that ranged from 1 to 4 $\mathrm{mm}$ Hg. Furthermore, recovery of ventilation after apnea preceded an increase in LES pressure in four of the eight piglets, 
as seen in Figure 4. When respiratory-related oscillations were visibly superimposed on baseline LES pressure, these oscillations disappeared during apnea, as seen in Figure 4.

\section{DISCUSSION}

The principal findings in this study are that hypoxia-induced increases in respiratory neural output and heart rate are associated with a significant increase in LES pressure, which is eliminated by atropine and, therefore, cholinergic in nature. To our knowledge, this is the first report demonstrating a significant effect of hypoxia-induced chemosensory stimulation on LES, linking changes in respiratory neural output with alteration in autonomic regulation of LES pressure. Of additional interest is the observation that development of apnea leads to a decline in LES pressure, suggesting a possible link between apnea and gastrointestinal reflux.

The ventilatory response to hypoxia has been previously characterized in anesthetized piglets, and the current responses of diaphragm amplitude and frequency are consistent with prior studies (12-15). A gradual increase in heart rate accompanied hypoxic exposure in these earlier studies $(12,13)$. In preterm human infants, the biphasic response of tidal volume to hypoxic exposure is almost identical to the response of diaphragm amplitude observed in the piglets. However, unlike our model, respiratory frequency decreases in preterm infants during hypoxic exposure (16). This discrepancy may be secondary to the relatively greater maturity of the piglet model. Nonetheless, we chose to study piglets because these studies could not be readily performed in human infants, and because ventilatory control has been well characterized in this animal model.

Our group has demonstrated that central parasympathetic motor output to the airways receives neural projections from the same region of the ventral medulla that is involved in respiratory control (1). Chemoreceptor stimulation by acute hypercapnia increased phrenic activity and elicited a cholinergically mediated contractile response of tracheal smooth muscle in piglets (4). Similarly, Waldron and Fisher (3) documented that hypercapnia significantly increased lung resistance in the newborn dog. Tay-Uyboco et al. (17) demonstrated that the elevated pulmonary resistance in infants with severe bronchopulmonary dysplasia is relieved by inhalation of $100 \%$ oxygen. Consistent with this observation, acute reduction in

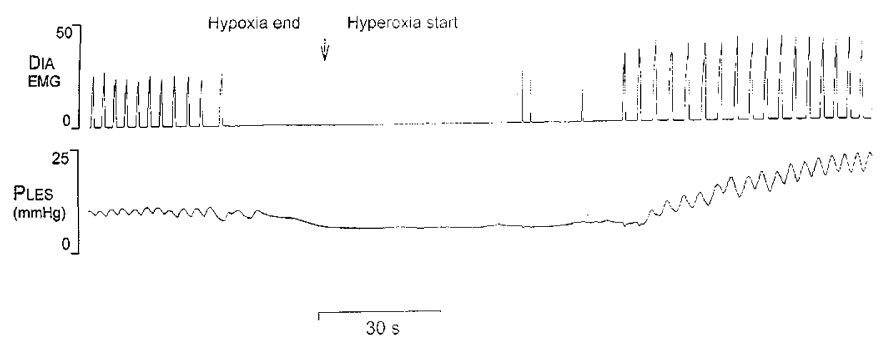

Figure 4. An example of apnea (after a second hypoxic exposure) that began before the end of hypoxia and was prolonged during reintroduction of $100 \%$ oxygen. Apnea was accompanied by a fall in baseline LES pressure (PLES) and a disappearance of phasic oscillations in LES pressure, both of which recovered with reinitiation of diaphragm (DIA) activity. inspired oxygen leads to airway constriction in infants with chronic lung disease (6). These observations led us to hypothesize that autonomic innervation of the LES might also be modulated by hypoxic exposure.

It is likely that the observed LES contraction induced by short-term hypoxia is initiated by stimulation of peripheral chemoreceptors from which impulses are sent via sinus nerve afferents to the nucleus tractus solitarius of the brainstem (18). At that site, signals are processed and then transmitted to LES-related vagal and/or sympathetic preganglionic neurons, and from these to the LES. Preganglionic parasympathetic motor innervation of the LES arises from the DMV and compact portion of the nucleus ambiguous. Stimulation of the DMV caudal to the area postrema relaxes the LES, probably via release of nitric oxide or vasoactive intestinal peptide; whereas activation of the rostral portion of the DMV evokes LES contraction (19) through the release of acetylcholine. Hence, the LES receives vagal efferent fibers that carry inhibitory and excitatory inputs. The findings of the present study suggest that the second-order nucleus tractus solitarius sensory neurons that receive excitatory inputs from carotid bodies project to the rostral portion of the DMV, because blockade of muscarinic receptors by atropine methylnitrate, a drug that does not cross the blood-brain barrier, inhibited the hypoxiainduced increase in LES pressure. This assumption on the role of cholinergic outflow in regulation of LES tone is supported by experiments showing that selective blockade of acetylcholine release from nerve endings innervating the LES in piglets inhibited resting LES tone (20).

Short-term hypoxic exposure may also effect LES tone by triggering a sympathetic chemoreflex, initiated by activation of peripheral chemoreceptors (21). More severe hypoxia may activate sympathetic outflow via direct effects on the brainstem (22). In both cases, the rostral ventrolateral medulla plays a critical role in these responses (23). Sympathetic innervation of the LES originates from the cardiac branch of the stellate ganglion, which joins the vagus nerve at the thoracic level as well as from the splanchnic nerve (24). These nonadrenergic fibers have an excitatory constrictor effect mediated through intramural cholinergic neurons $(25,26)$. Hence, the present results cannot rule out a contribution from sympathetic outflow to the increase of LES tone produced by stimulation of arterial chemoreceptors.

The diaphragm is composed of a costal part, which inserts into the ribs, and a crural part, which is attached to the vertebral column. The crural diaphragm forms a canal through which the esophagus enters the abdomen. Enhanced contraction of the crural diaphragm may have contributed to the hypoxia-induced increase in LES tone (27). There is ample evidence in animal and human studies that contraction of the crural diaphragm increases pressure at the esophagogastric junction $(27,28)$. However, in our study, this increase in LES pressure was abolished by muscarinic blockade without affecting the diaphragmatic response, suggesting a central origin for this association. It is possible that the oscillations in LES pressure that were in phase with the respiratory cycle, and variably observed between piglets (as seen in Figs. 3 and 4), were related to phasic crural diaphragm contraction. We have previously ob- 
served in piglets that stimulation of peripheral chemoreceptors increases cholinergic outflow to the most distal airways. With the onset of phrenic firing, airway and alveolar pressures became phasically modulated (5). This suggests that an increase in cholinergic motor activity to airways or LES arises from an elevated activity of vagal preganglionic neurons, which is affected by changes in respiratory drive.

In this study, we observed the development of apnea in eight of 12 piglets. These apneic episodes were precipitated by peripheral chemoreceptor inhibition induced by the subsequent hyperoxic exposure. In the three of these piglets, who became apneic after a second hypoxic exposure, hypoxic depression may also have contributed to the development of apnea (13, 14). Apnea preceded a decrease in LES pressure, and subsequent recovery of ventilation occurred before an increase in LES pressure in four of the eight piglets. These findings suggest that loss of respiratory neural output might contribute to loss of LES tone. This is consistent with the observation that cholinergically mediated tracheal tone depends on the level of respiratory drive in cats (29). This is also consistent with recent clinical data that examined the temporal relationship between gastroesophageal reflux and apnea in infants with intermittent episodes of reflux and a history of apparent life-threatening events. Although the majority of apnea was not temporally related to episodes of reflux, when a relationship was observed, apnea preceded rather than followed episodes of reflux (11). This is entirely consistent with the recent clinical observation that antireflux medications do not reduce the frequency of apnea in preterm infants (10).

There are some limitations to this study that merit discussion. In preterm human infants, transient LES relaxation appears to be the predominant mechanism for inducing reflux (30). However, there is a paucity of information regarding the anatomy and physiology of the LES in the pig that make it difficult to assess the role of immaturity on our findings, and do not allow us to extrapolate these observations in anesthetized piglets to human infants. Additionally, we cannot characterize the precise contributions of parasympathetic and sympathetic pathways to the observed response, although we have identified a definitive role for cholinergic transmission in the LES responses to hypoxia. It is possible that the response of the LES to hypoxia might have arisen from altered baroreceptor activity, as has been reported for airway caliber (31). However, we observed no significant effect of hypoxic exposure on blood pressure at $3 \mathrm{~min}$. We recognize that both sedation and anesthesia, as used in this study, may reduce respiratory neural output as well as cholinergic responses, such as those of the LES, to chemoreceptor stimulation. This should not have influenced the major finding from this study that hypoxia induced parallel increases in lower esophageal tone and respiratory output. We also acknowledge that the hyperoxic state used before hypoxic exposure differs from the usual clinical status in infants. However, this was done to eliminate interanimal differences in baseline $\mathrm{Po}_{2}$ and peripheral chemoreceptor function. Finally, it is important to recognize that our model differs from the clinical situation where a decrease in neural output with apnea is followed by hypoxia, a sequence of events we were not able to simulate in this protocol.
We conclude that hypoxia-induced increase in respiratory neural output is associated with a cholinergically mediated enhancement of LES constriction. Recognizing the limitations in extrapolating from our animal model to the clinical situation, we speculate that, in the presence of increased respiratory neural output, hypoxia is unlikely to provoke gastroesophageal reflux in infants unless there is an associated decrease in respiratory neural output, as occurs during spontaneous apnea.

\section{REFERENCES}

1. Haxhiu MA, Jansen AS, Cherniack NS, Loewy AD 1993 CNS innervation of airway-related parasympathetic preganglionic neurons: a transneuronal labeling study using pseudo rabies virus. Brain Res 618:115-134

2. Haxhiu MS, Erokwu BO, Cherniack NS 1996 The brainstem network involved in coordination of inspiratory activity and cholinergic outflow to the airways. J Auton Nerv Syst 61:155-161

3. Waldron MA, Fisher JT 1988 Differential effects of $\mathrm{CO}_{2}$ and hypoxia on bronchomotor tone in the newborn dog. Respir Physiol 72:271-282

4. Haxhiu-Poskurica B, Carlo WA, Miller MJ, DiFiore JM, Haxhiu MA, Martin RJ 1991 Maturation of respiratory reflex responses in the piglet. J Appl Physiol 70:608-616

5. Martin RJ, Dreshaj IA, Miller MJ, Haxhiu MA 1995 Neurochemical control of tissue resistance in piglets. J Appl Physiol 79:812-817

6. Teague WG, Pian MS, Heldt GP, Tooley WH 1988 An acute reduction in the fraction of inspired oxygen increases airway constriction in infants with chronic lung disease. Am Rev Respir Dis 137:861-865

7. Hornby PJ, Abrahams TP 2000 Central control of lower esophageal sphincter relaxation. Am J Med 108:90S-98S

8. Menon AP, Schefft GL, Thach BT 1985 Apnea associated with regurgitation in infants. J Pediatr 106:625-629

9. de Ajuriaguerre M, Radvanyi-Bouvet MF, Huon C, Moriette G 1991 Gastroesophageal reflux and apnea in premature born infants during wakefulness and sleep. Am J Dis Child 145:1132-1136

10. Kimball AL, Carlton DP 2001 Gastroesophageal reflux medications in the treatment of apnea in premature infants. J Pediatr 138:355-360

11. Arad-Cohen N, Cohen A, Tirosh E 2000 The relationship between gastroesophageal reflux and apnea in infants. J Pediatr 137:321-326

12. Elnazir B, Marshall JM, Kumar P 1996 Postnatal development of the pattern of respiratory and cardiovascular response to systemic hypoxia in the piglet: the roles of adenosine. J Physiol 493:573-585

13. Miller MJ, Haxhiu MA, Haxhiu-Poskurica B, Dreshaj IA, DiFiore JM, Martin RJ 2000 Recurrent hypoxic exposure and reflex responses during development in the piglet. Respir Physiol 123:51-61

14. Moss IR 2000 Respiratory responses to single and episodic hypoxia during development: mechanisms of adaptation. Respir Physiol 121:185-197

15. Suguihara C, Bancalari E, Hehre D, Duara S, Gerhardt T 1994 Changes in ventilation and oxygen consumption during acute hypoxia in sedated newborn piglets. Pediatr Res 35:536-540

16. Martin RJ, DiFiore JM, Jana L, Davis RL, Miller MJ, Coles SK, Dick TE 1998 Persistence of the biphasic ventilatory response to hypoxia in preterm infants. J Pediatr 132:960-964

17. Tay-Uyboco JS, Kwiatkowski K, Cates DB, Kavanagh L, Rigatto H 1989 Hypoxic airway constriction in infants of very low birth weight recovering from moderate to severe bronchopulmonary dysplasia. J Pediatr 115:456-459

18. Finley JCW, Katz DM 1992 The central organization of carotid body afferent projections to the brainstem of the rat. Brain Res 572:108-116

19. Rossiter CD, Norman WP, Jain M, Hornby PJ, Benjamin S, Gillis RA 1990 Control of lower esophageal sphincter pressure by two sites in dorsal motor nucleus of the vagus. Am J Physiol 259:G899-G906

20. Pasricha PJ, Ravich WJ, Kalloo AN 1993 Effects of intrasphincteric botulinum toxin on the lower esophageal sphincter in piglets. Gastroenterology 105:1045-1049

21. Smith ML, Muenter NK 2000 Effects of hypoxia on sympathetic neural control in humans. Respir Physiol 121:163-171

22. Cherniack NS, Ernsberger P, Mitra J, Haxhiu MA 1995 The role of the ventral medulla in hypoxic respiratory depression and sympathetic excitation. In: Trouth CO, Millis RM, Kiwull-Schöne, Schläfke ME (eds) Ventral Brainstem Mechanisms and Control of Respiration and Blood Pressure. Marcel Dekker, New York, pp 103-129

23. Guyenet PG 2000 Neural structures that mediate sympathoexcitation during hypoxia. Respir Physiol 121:147-162

24. Gonella J, Niel JP, Roman C 1979 Sympathetic control of lower oesophageal sphincter motility in the cat. J Physiol 287:177-190

25. Gonella J, Niel JP, Roman C 1980 Mechanism of the noradrenergic motor control on the lower oesophageal sphincter in the cat. J Physiol 306:251-260

26. Fournet J, Snape Jr WJ, Cohen S 1979 Sympathetic control of lower esophageal sphincter function in the cat. Action of direct cervical and splanchnic nerve stimulation. J Clin Invest 63:562-570

27. Mittal RK, Balaban DH 1997 The esophagogastric junction. N Engl J Med 336:924932 
28. Martin CJ, Dodds WJ, Liem HH, Dantas RO, Layman RD, Dent J 1992 Diaphragmatic contribution to gastroesophageal competence and reflux in dogs. Am J Physiol 263:G551-G557

29. Haxhiu MA, Deal EC, Cherniack NS 1989 Influence of respiratory drive on airway responses to excitation of lung C-fibers. J Appl Physiol 67:203-209
30. Omari TI, Benninga MA, Barnett CP, Haslam RR, Davidson GP, Dent J 1999 Characterization of esophageal body and lower esophageal sphincter motor function in the very premature neonate. J Pediatr 135:517-521

31. Schultz HD, Pisarri TE, Coleridge HM, Coleridge JCG 1987 Carotid sinus baroreceptors modulate tracheal smooth muscle tension in dogs. Circ Res 60:337-345 